\title{
Programming Realized Based on the Block Model for Grounding Grids Corrosion Diagnosis
}

\author{
Cheng Hongli, a , Zhang Kuaikuai, ${ }^{2, b}$, Yang zhao ${ }^{3, c}$ \\ ${ }^{1,2,3}$ Xi'an University of Science \& Technology, Xi'an, China \\ a,b991811551@qq.com, c1158979801@qq.com
}

Keywords: Grounding grids, Corrosion diagnosis, Block model , Software

\begin{abstract}
In order to solve the problem of corrosion diagnosis of grounding grids, related program modules of modeling and corrosion diagnosis of grounding grids is developed based on the block model on the platform of Matlab. The programming to realize the block model of a grounding grids is described. The programming of automatically establishment of quasi-blocks, divided into blocks, the judgment of block kinds, the judgment of unit's autonomy performance, determine the set of considered intrinsic branches is detailed. The programming of all branch resistances diagnosis form intrinsic grounding grids to actual grounding grids is analyzed. An experiment about a 60 branches grounding grids corrosion diagnosis is completed. The adopted approach is shown feasible.
\end{abstract}

\section{Introduction}

The grounding grids corrosion diagnosis method is mainly divided into electromagnetic field analysis method and circuit theory analysis method. The former is mainly to find the corrosion branches' location of the grounding grids[1].The latter is a typical analog circuit corrosion diagnosis method, mainly including: calculate each conductor's actual value of grounding grids through the Tellegen's theorem resistance measurement method[2]; establish node voltage equation and use simplex method solve each conductor's resistance increment[3]; significant increase the equations number by using the method of multiple measurements of each incentive, and obtain the best solution of grounding grids branch resistance in the sense of least square by using iterative method to solve the node voltage equation; and the corresponding corrosion diagnosis software system of grounding grids [4].

The grounding grid usually need comprehensive test in the existing diagnosis method. Each branches also need corrosion diagnose. The method through iterative operating the large-scale corrosion diagnosis equation describing the whole grounding grid diagnose each branch's resistance value. The calculate efficiency is low, measurement error cross interference is existed. Sometimes, a large measurement error will produce significant effect to the corrosion diagnosis of all the branches. According to the relationship between the branches, a grounding grid corrosion diagnosis method based on the block model is presented[5]. The calculate efficiency is improved, the impact of the error cross interference is reduced and the nonlinear iteration is avoided in solving the intrinsic branch.

In order to diagnose grounding grid block, on the basis of the existing literature[6-7],the method of establishing the grounding grid block model is proposed. All kinds of grounding grid block corrosion diagnosis are analyzed. A method by using the rule, formula and numerical judgment is proposed. The branch is diagnosed by tracing back step by step dichotomy method. The grounding block corrosion diagnosis software is developed based on the platform of Matlab. 


\section{Program realized of grounding grid block}

\section{Basic flow}

According to the range of diagnosing branch, in quasi-block, grounding grids is divided into different independent blocks. According to the projection of these blocks, the related section in the corresponding intrinsic grounding grids is determined. In the block corresponding intrinsic grounding grids, each section public node is tested. According to the test data, the solving intrinsic branch set is solved. The software chart of the grounding grids block model is divided into the following steps:

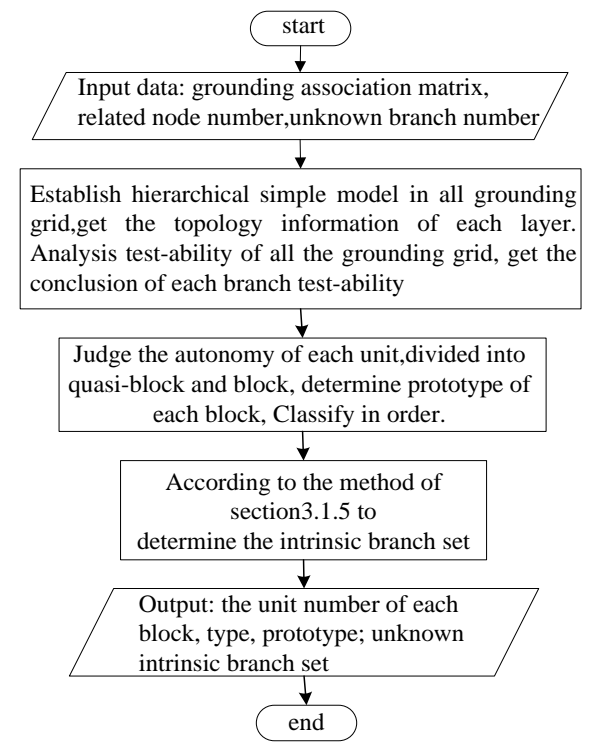

Fig.1 Software flow chart on block model

(1)Input.Read the node branch correlate matrix of grounding grids' topology,read the distribution of nodes,and read the scope of diagnosis.

(2)Establish layered simple model ${ }^{[6]}$, and analysis test ability[5]. Obtain each layer topology information and the conclusion of all branches test-ability.

(3)Divided into quasi-block through combining units where related element networks exist.

(4)Judge unit autonomy[8], divide into blocks and determine the prototype of every block , classify the blocks, and determine the set of considered intrinsic branches.

The software flow chart on block model is shown in fig.1.

\section{Quasi-blocks division}

The further research on grounding grids show that some branches are interrelated, the resistance must solve together not separated. In grounding grids, the set consist of some correlating branches is called quasi-block. Each layer topology information and conclusion of branch test-ability is obtained. And the data is input, parallel relationship between the element networks is cycle compared. If existing parallel, the element network is belong to the same block. Divided into quasi-block and get correlation matrix is obtained. Software flow chart on quasi-blocks is shown in fig. 2.

\section{Divide the block and determine prototype}

Block is a subset of the quasi-blocks, the divided principle follows literature [5].

Basic on literature [9], this paper put forward a new proposal about unit autonomous judgment: if a element network is clear, the corresponding block is not uncertain clear. That means it is not certain unit. The judgment has joined in the program.

According to the said principle to divide block, the branch of each block prototype is clear. To do so, the block corrosion diagnosis process will be convenient. A few blocks of a quasi-block can have public branch. The software flow chart on dividing into block is shown in figure 3 .

\section{Projection and determine the unknown intrinsic branch set}

According to the node partition method[9], the unknown intrinsic branch set and block projection is determined, basic steps of the program are as follows:

(1) Manual input to test public node set: node-set. 
(2) According to the intrinsic branch correlation matrix, all related nodes are obtained, node partition. Unknown intrinsic branch set of each partition is determined.

(3) According to the linear method proposed in literature [9] solving the intrinsic branch resistance,the corresponding intrinsic branch is calculated by function (lsqlin) in Matlab and ranked in order. The value of branches not belong to range is 0 .

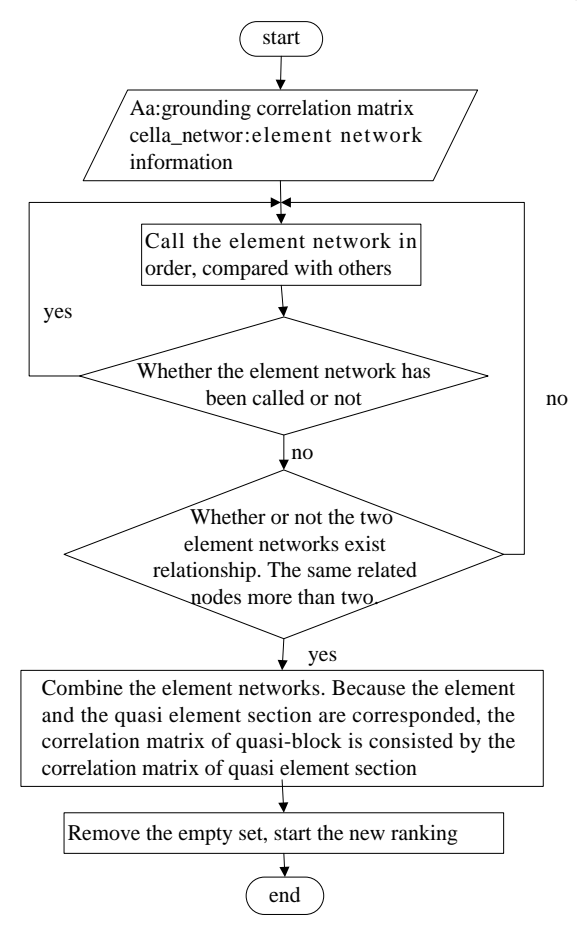

Fig.2 Software flow chart on quasi-blocks

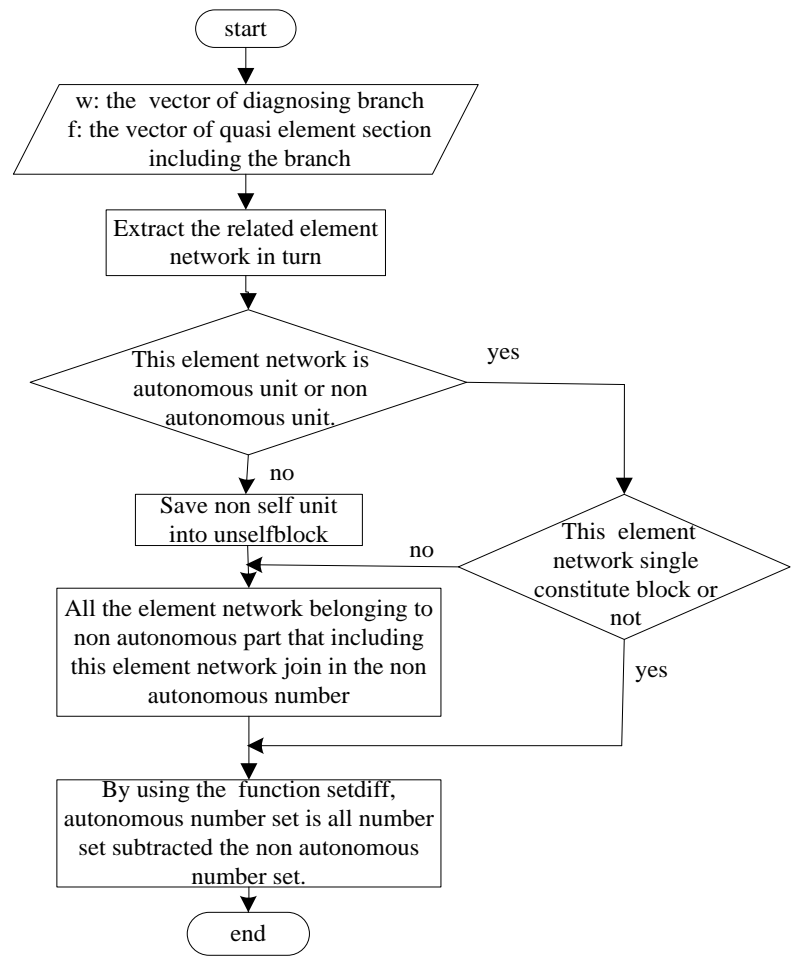

Fig.3 Software flow chart on dividing into blocks

\section{Blocks corrosion diagnosis program}

\section{State test-ability analyzing rules}

State test-ability rules:

(1) If the branch resistances in intrinsic grounding grids are normal, the branch resistances in actual grounding grids can be respectively diagnosed as the corresponding design value, and each branch is transformed into defining.

(2) If an intrinsic branch resistance is diagnosed to normal, the resistances of all branches between the node $i$ and $\mathrm{j}$ in grounding grids are normal value.

(3) If the branches' resistances in an element network are diagnosed to normal, the resistances of all branches in corresponding element section are respectively equal to its normal value.

(4)If a branch resistance in a element section is normal, the related branch resistance of the actual grounding grids included in corresponding quasi element section is respectively equal to normal value.

The diagnosis result of the intrinsic branch resistance in the unknown intrinsic branch set is obtained through tracing back step by step method and the proposed rules.

The computational complexity can be greatly reduced through using rules.

\section{Corrosion diagnosis of uncertain branch}

In the basis of the diagnosis of intrinsic branch resistance and all defining element network branch resistance, the uncertain branch resistance range can be solved through dichotomy search method. The software flow chart is shown in fig. 4. 


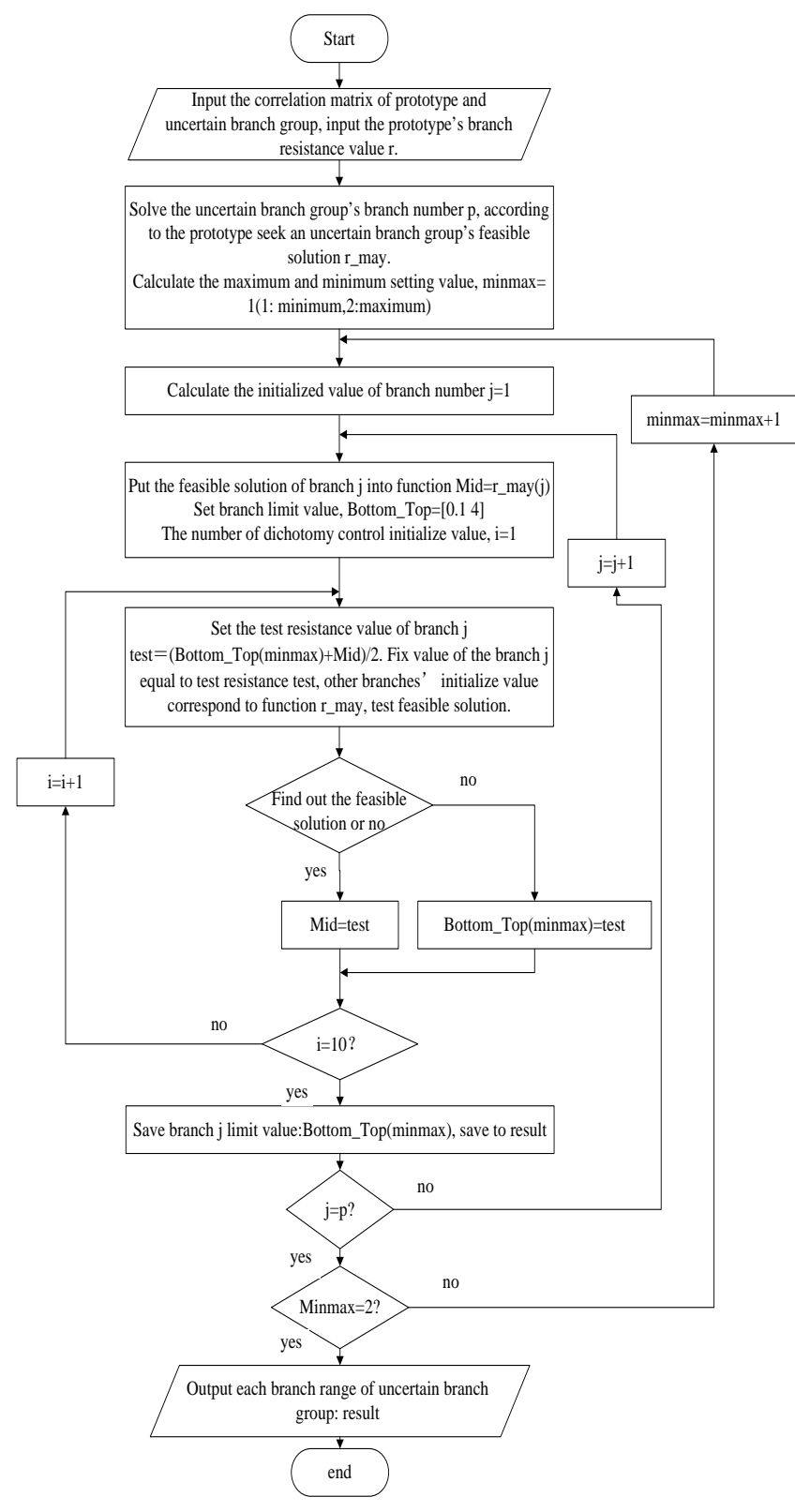

Fig.4 Flow chart on getting resistances of uncertain branch

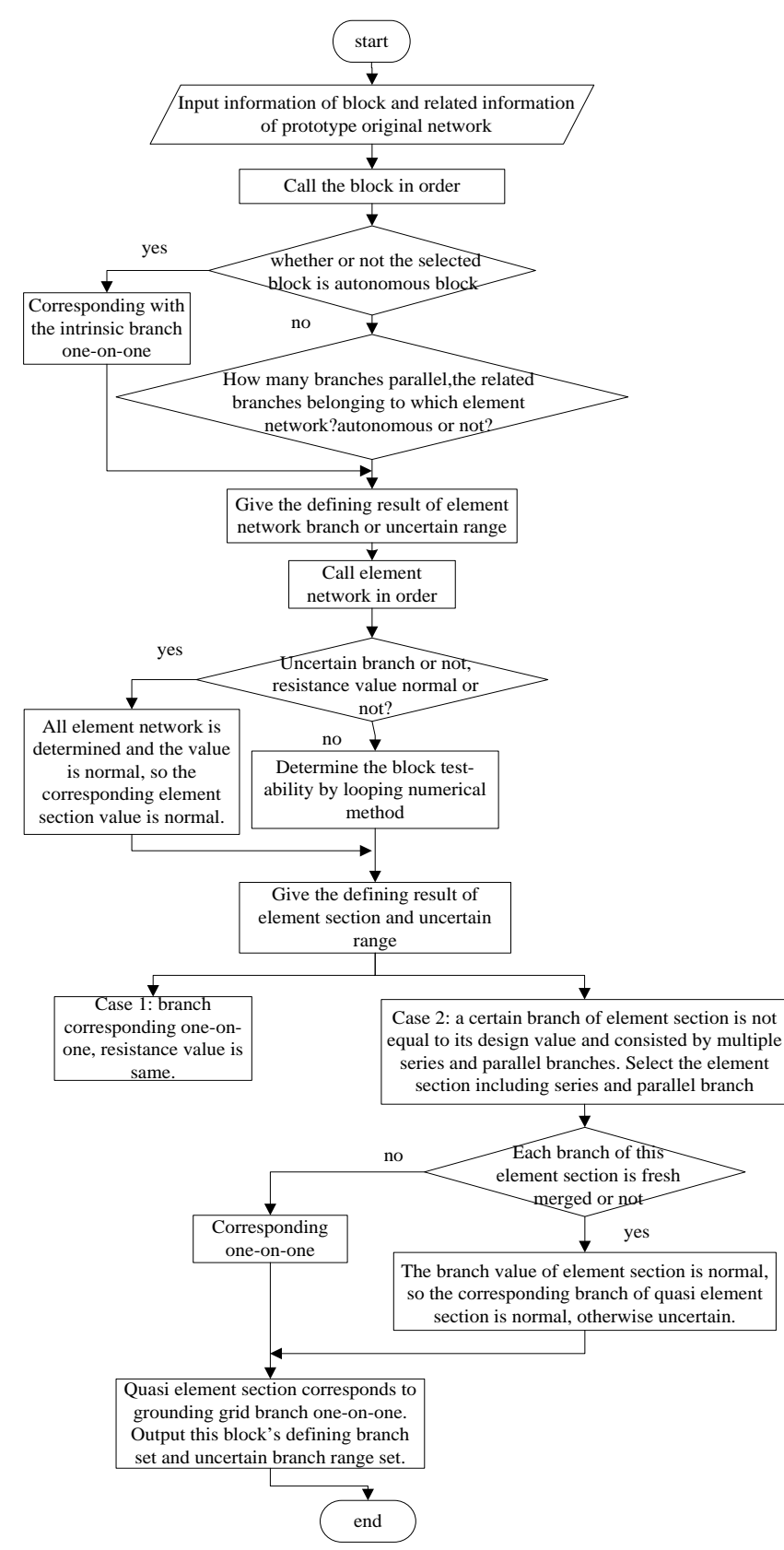

Fig.5 Software flow chart on corrosion diagnosis of defining branch

In this designing program, uncertain branch groups' corrosion diagnosis is only a sub module program and can be called at any time. Its determination and divination are provided by the defining branch corrosion diagnosis.

\section{Defining branch corrosion diagnosis}

Judge the type of the selected block

Call the related block in order. If there are abnormal branches or uncertain branches judging through the section2.1 method, the next step is needed. The selected blocks are divided into 4 types:

(1) Defining block. All branches of actual grounding grids in this block are defined.

(2) Quasi defining block. All element sections in this block are defined. But quasi element section and element section are connected through series and parallel. It is belong to the second class uncertain branch groups. The actual grounding grids branch range can be got by calling section 2.2 subroutine.

(3)Uncertain block. All element sections in this block are uncertain. It is belong to the first uncertain branch groups. The actual grounding grids branch range is obtained by calling section 2.2 subroutine and skipping the process tracing back step by step. 
(4) Mixed block. The element section branches in this block include both contain and uncertain. It is belong to the third class uncertain branch groups. After the complete defining corrosion diagnosis, the section 2.2 subroutine is called.

Three processes of tracing back step by step

\begin{tabular}{ccccccccccccc} 
Branch number & $R_{(5,11)}$ & $R_{(10,11)}$ & $R_{(11,12)}$ & $R_{(10,16)}$ & $R_{(11,17)}$ & $R_{(12,18)}$ & $R_{(15,16)}$ & $R_{(16,17)}$ & $R_{(17,18)}$ & $R_{(15,21)}$ & $R_{(16,22)}$ & $R_{(17,23)}$ \\
\hline Actual value & 0.2244 & 0.1020 & 0.1063 & 0.1025 & 0.1046 & 0.4374 & 0.3626 & 1.3099 & 0.1002 & 0.5342 & 1.3027 & 0.7184 \\
Diagnosis value & 0.2033 & 0.1101 & 0.1082 & 0.0988 & 0.1023 & 0.4199 & 0.3682 & 1.3504 & 0.0939 & 0.5234 & 1.3541 & 0.7325 \\
Branch number & $R_{(18,24)}$ & $R_{(20,21)}$ & $R_{(21,22)}$ & $R_{(22,23)}$ & $R_{(23,24)}$ & $R_{(20,26)}$ & $R_{(21,27)}$ & $R_{(22,28)}$ & $R_{(23,29)}$ & $R_{(24,30)}$ & $R_{(25,26)}$ & $R_{(26,27)}$ \\
\hline Actual value & 0.5317 & 1.3361 & 0.3530 & 0.5214 & 0.1016 & 0.1005 & 0.2244 & 0.1015 & 0.4665 & 0.1024 & 0.0999 & 0.1018 \\
Diagnosis value & 0.5195 & 1.2619 & 0.3581 & 0.4876 & 0.0984 & 0.0973 & 0.1083 & 0.1053 & 0.4411 & 0.1055 & 0.1113 & 0.0971 \\
Branch number & $R_{(27,28)}$ & $R_{(28,29)}$ & $R_{(29,30)}$ & $R_{(26,32)}$ & $R_{(27,33)}$ & $R_{(28,34)}$ & $R_{(29,35)}$ & $R_{(30,0)}$ & $R_{(32,33)}$ & $R_{(33,34)}$ & $R_{(34,35)}$ & $R_{(35,0)}$ \\
\hline Actual value & 0.5456 & 0.0953 & 0.3582 & 0.2314 & 0.5238 & 0.1001 & 0.4222 & 1.3155 & 0.3460 & 0.0974 & 0.4285 & 0.1015 \\
Diagnosis value & 0.5488 & 0.7618 & 0.3382 & 0.2200 & 0.5482 & 0.1042 & 0.4102 & 1.3872 & 0.2222 & 0.0998 & 0.3776 & 0.4068
\end{tabular}

Table.1 Result of diagnosis on defining branch

In diagnosing the grounding grids clear branch in a block, the branch resistance in block prototype is obtained through the method tracing back step by step and the formula proposed in literature [6]. The program flow chart is shown in Fig.5.

\section{Examples}

The results of grounding grids block corrosion diagnosis program is explained in the experiment about 60 branches grounding grids shown in Fig.6 (a). In Fig.6 (a), digital represents node's number, and solid black dots represent related nodes. The grounding grids related nodes are: 3, 5, 8, 10, 12, 13, $15,17,18,20,21,22,23,24,25,27,29,30,32,33,34$ and 0.The block model is shown in Fig.6 (b).The shadow area of Fig.6 (a) represents the range of corrosion diagnosis.

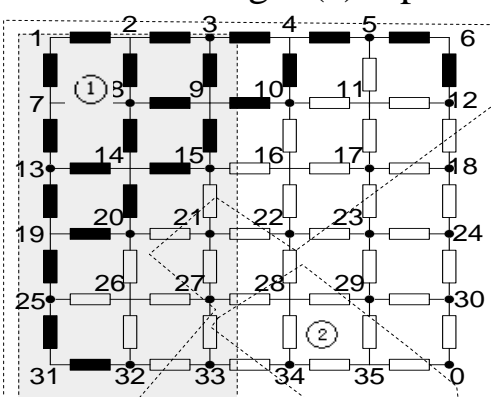

(a)selected branch and quasi-bločk diagram of a 60 branch experiment grounding grids

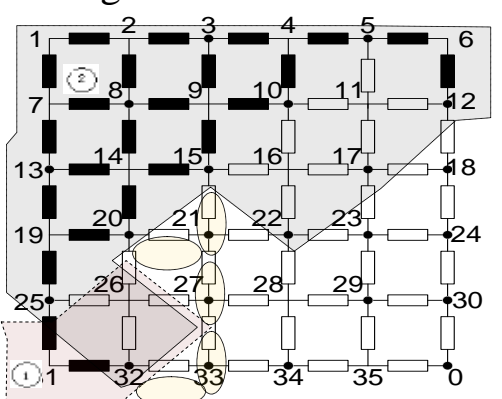

(b)7 divided blocks of the proposed corrosion diagnosis area

Fig.6 Block model of grounding grids

The result is much close to the result of literature [7] methods. But comprehensive test and calculation of grounding grids is not needed. The large-scale grounding grids are divided into 7 small sub networks. Large matrix operation is avoided and calculation efficiency is improved. The result of diagnosis on defining branch is shown in Table.1.

\section{Conclusions}

Experiment result shown that block model program of grounding grids in this paper is feasible. The corrosion diagnosis of grounding grids is more convenient. According to the block prototype, the range of defining branch resistance and uncertain branch resistance is obtained. This divided block method splits grounding grids into several small-scale networks. Combining with the principle of state test-ability analysis, amount of calculation is greatly reduced and the solving efficiency is improved. 


\section{References}

[1] Dawalibi F P. Electromagnetic Fields Generated by Overhead and Buried Short Conductors Part 2:Ground Conductor [J].IEEE Trans Power Delivery,1986,11:(11)2-119.

[2] Hu Jun, Zhang Rong, He Jin-liang, etal. Novel Method of Corrosion Diagnosis for Grounding Grids [C].Proceedings of the 2000 International Conference on Power System Technology, Perth, Australia, 2000,3:1365-1370.

[3] Zhang Xiaoling, Huang Qingyang. Fault Diagnosis of Grounding Grid of Electric Power Plants and Sub Stations[J]. Proceedings of the CSU-EPSA, 2002,14 (1):48-51.

[4] Huang Wenwu, Wen Xi Shan, Zhu Zhengguo. Development of the Diagnosis Software System for the Corrosion and Broken Point of the Grounding Grid[J].High voltage engineering,2005,31 (7): 42-44.

[5] LiuJian, Ni Yunfeng, Wang Sen, etal. Grounding Grids Corrosion Diagnosis Using a Block Dividing Approach [J]. High voltage engineering, 2011,37 (5): 1194-1202.

[6] LiuJian, Wang Shuqi,Li Zhizhong, etal. Test-ability of Grounding Grids Corrosion Diagnosis[J]. High voltage engineering, 2008,34 (1):32-36.

[7] Wang Shuqi,Ni Yunfeng, Liu Jian, et al. The Topology Analysis Algorithm in Corrosion Diagnosis of Grounding Grids[J]. microelectronics \& computer, 2008,25 (4):13-17.

[8] Wang Shuqi, Liu Jian, et al. Hybrid Approach Based on Rules and Numerical Methodology to Evaluate Test-ability of Branches of Grounding Grid[J]. High voltage engineering, 2008,34 (9): 1964-1970

[9] Ni Yunfeng, Liu Jian, Lu Wei, et al. Block Model of Grounding Grid and Its Programming[J]. Computer engineering and applications, 2009,18 (6): 62-65. 